\title{
An implicit free surface algorithm for geodynamical simulations
}

\author{
Stephan C. Kramer ${ }^{\mathrm{a}, \mathrm{b}, 1}$, Cian R. Wilson ${ }^{\mathrm{c}}$, D. Rhodri Davies ${ }^{\mathrm{a}}$ \\ ${ }^{a}$ Department of Earth Science and Engineering, Imperial College London, SW7 2AZ, UK \\ ${ }^{b}$ Institute of Shock Physics, Imperial College London, SW7 2AZ, UK \\ ${ }^{c}$ Lamont-Doherty Earth Observatory, Columbia University, Palisades, New York 10964, USA
}

\begin{abstract}
Identifying the dominant controls on Earth's surface topography is of critical importance to understanding both the short- and long-term evolution of geological processes and past- and present-day dynamics of Earth's coupled mantle-lithosphere system. The ability to simulate a stress free - or a so-called 'free surface' - boundary condition is required to examine such processes via numerical models. However, at present, geodynamical models incorporating a free surface are limited, as most underlying free surface algorithms place severe restrictions on the computational timestep. Consequently, the simulations are often intractable. In this study, we introduce a new approach for incorporating a free surface within geodynamical models: an algorithm, in which free surface elevation is treated as an independent variable and is solved for in conjunction with the momentum and continuity equation, using implicit time integration. We demonstrate that the method is straightforward to implement in existing models and, using a series of analytical and benchmark comparisons, we show that it does not suffer from the timestep constraints of previous schemes. Furthermore, the scheme can be made second order accurate in time, at no additional cost. The method therefore dramatically improves the computational efficiency of geodynamical simulations including a free surface, whilst maintaining solution accuracy.
\end{abstract}

Keywords: Free surface, Topography, Dynamics of lithosphere and mantle, Tectonics and landscape evolution, Numerical methods and analysis

\section{Introduction}

While the majority of Earth's present-day surface topography results from crustal and lithospheric thickness variations, generated by the movement of tectonic plates along Earth's surface, there is mounting evidence to suggest that a significant proportion results from viscous stresses created by flow within Earth's mantle. Although such dynamic topography is transient, it can exert a substantial influence upon surface processes (Braun, 2010); it has, for example, played a role in the geological evolution of the North American continent (Liu et al., 2008; Spasojevic et al., 2009), the establishment of Amazon drainage patterns (Shephard et al., 2010) and in controlling the overflow of North Atlantic Deep Water across the Greenland-Scotland Ridge (Wright and Miller, 1996; Poore et al., 2009). In turn, surface processes, such as erosion of topography and deposition of eroded sediments at oceanic trenches, may actively influence patterns of deformation, affecting plate motions and mantle flow (e.g.

${ }^{*}$ Corresponding author: s.kramer@imperial.ac.uk 
Koons, 1990; Molnar and England, 1990; Beaumont et al., 1996; Willett, 1999; Beaumont et al., 2001; Braun, 2006, 2010; Kaus et al., 2008; Iaffaldano et al., 2006, 2011).

Key to an improved understanding of the interactions between surface processes and mantle and lithosphere dynamics are numerical models of this coupled system. However, to model such interactions in a self-consistent manner, one must take into account Earth's free surface, which has, thus far, proved challenging. Several distinct methods are currently used to simulate surface topography in geodynamical models. Dynamic topography may be derived from the normal stress at a free slip boundary (Hager et al., 1985; McKenzie, 1977; Zhong et al., 1993). This approach however ignores the timescales involved in the response of surface topography to changes in the interior. In studies by Zhong et al. (1996) and Gurnis et al. (1996) it is shown how the history-dependent free surface evolution can be taken into account by adjusting the normal stress boundary condition, to include topographic stresses based on the evolution of free surface height calculated in the previous timestep. This method modifies the boundary condition at the surface in an unchanged Eulerian mesh.

Methods that do move the mesh to follow topography include fully Lagrangian schemes (e.g. Melosh and Raefsky, 1980; Lavier et al., 2000), in which all calculations are done on a Lagrangian mesh that follows the flow velocity, and Arbitrary Lagrangian-Eulerian (ALE) methods (e.g. Fullsack, 1995) that employ a mesh that does not necessarily move with the flow velocity everywhere. For these methods a zero normal stress boundary condition can be applied naturally at the moving free surface. Other free surface modelling approaches include: using marker chain or level set functions to track the free surface on a background Eulerian mesh (e.g. Harlow and Welch, 1965; Muhlhaus, 2007); and the 'sticky-air' approximation, where a low viscosity, low density layer is placed above a free slip boundary, thus allowing the material interface to behave similarly to a free surface (Schmeling et al., 2008; Crameri et al., 2011).

While each method has its own advantages and disadvantages (for a detailed discussion see Crameri et al., 2011), most approaches pose limitations on the computational timestep. To ensure numerical stability in free surface simulations, the computational timestep must often be at least an order of magnitude less than the timestep in an identical simulation employing a free slip boundary. This is due to the temporally explicit coupling between free surface movement, which is computed at a previous timestep, and the resulting topographic stresses; an upward velocity at the free surface does not feel the increasing (decreasing) load from rising (falling) topography until the next timestep. As a consequence, with current methods, free surface simulations are computationally expensive, and are generally considered intractable for global mantle convection models (e.g. Bunge et al., 2002; Tackley, 2008; Zhong et al., 2008; Wolstencroft et al., 2009; Davies and Davies, 2009; Styles et al., 2011).

In an attempt to overcome this issue, Kaus et al. (2010) introduce a free surface stabilization approach, which adds stabilization terms to the momentum equation, mimicking the feedback of changing topography on the underlying dynamics. Such an approach allows for larger timesteps with free surface simulations and maintains solution accuracy. An alternative approach, which appears to have similar benefits (Fuchs et al., 2011), is presented by Schmeling et al. (2008), based upon a 4th order Runge Kutta scheme combined with a predictor corrector step for advecting markers. In this paper, we introduce a new free surface method, equally applicable to both Eulerian and Lagrangian models, in which free surface elevation is treated as an independent variable that is solved for in conjunction with 
the momentum and continuity equation, using implicit time integration. This removes the timestep constraint without the need for additional stabilization terms. Moreover, we show that the method can be made second order accurate in time at no additional cost, where previous methods are only first order accurate. The method therefore dramatically improves computational efficiency, allowing for far larger timesteps than explicit free surface methods, whilst maintaining solution accuracy.

The structure of the paper is as follows: we begin by presenting an overview of our methodology, including the governing equations and boundary conditions, where the boundary conditions are dealt with in a manner similar to Zhong et al. (1996). We then demonstrate that the solution strategy employed for an implicit free surface treatment requires only a minor modification from our standard solution strategy for velocity and pressure, as implemented in the numerical model Fluidity (Davies et al., 2011), which we use throughout this paper. An explicit free surface method is also described, as we use this for comparative purposes. We next present a series of test cases, including analytical and benchmark comparisons, which demonstrate the computational efficiency and physical accuracy of our method. We end by discussing the advantages of the method and its potential use in large-scale geodynamical models.

\section{Methodology}

\subsection{Equations}

To explain the boundary conditions employed at the free surface, we start from the Stokes equations with a pressure that includes the hydrostatic component. Consider a density distribution $\rho=\rho_{0}+\rho^{\prime}$, where $\rho_{0}$ is the reference density and $\rho^{\prime}$ a perturbation density. The incompressible Stokes equations forced by a corresponding gravity term are given by:

$$
\begin{array}{r}
-\sum_{j} \partial_{j} \hat{\sigma}_{i j}=-\left(\rho_{0}+\rho^{\prime}\right) g k_{i}, \\
\sum_{i} \partial_{i} u_{i}=0,
\end{array}
$$

with velocity $u_{i}$, stress tensor $\hat{\sigma}_{i j}=\tau_{i j}-\hat{p} \delta_{i j}$, where $\hat{p}$ is the full pressure and $\tau_{i j}$ the deviatoric stress tensor, gravitational acceleration $g$ and $k_{i}$ a unit vector in the upward direction. The deviatoric stress tensor is given by:

$$
\tau_{i j}=\mu\left(\partial_{i} u_{j}+\partial_{j} u_{i}\right),
$$

where $\mu$ is the dynamic viscosity. These equations are considered together with the following boundary conditions:

No slip: $u_{i}=0$,

Free slip: $\left\{\begin{aligned} \sum_{i} u_{i} n_{i} & =0, \\ \sum_{i, j} n_{i} \tau_{i j} t_{j} & =0,\end{aligned}\right.$ 


$$
\text { Free surface: }\left\{\begin{aligned}
\sum_{i} n_{i} u_{i} & =\frac{\partial \eta}{\partial t} \sum_{i} n_{i} k_{i}, \\
\sum_{j} n_{j} \hat{\sigma}_{i j} & =0 .
\end{aligned}\right.
$$

Here, $n_{i}$ is the outward pointing normal vector to the boundary and $t_{i}$ any tangential vector. At the free surface, the kinematic boundary condition (5a) relates the normal velocity to the movement of the free surface. This movement is given by the time-derivative of the free surface elevation $\eta$, which measures the distance, in the upward direction, between a fixed reference plane $z=z_{0}$ and the free surface at $z=z_{0}+\eta$. The inner product $\sum_{i} n_{i} k_{i}$ takes into account potential different directions of this normal velocity and the upward direction $k_{i}$. Indeed, at the core-mantle-boundary, these are opposite and the inner product becomes negative.

\section{Removal of hydrostatic pressure}

A common step when solving equations (1) - (5) is to subtract the main hydrostatic component from the full pressure $\hat{p}$, and solve in terms of a perturbation pressure $p=$ $\hat{p}-\rho_{0} g\left(z_{0}-z\right)$, where $z$ is defined as positive upwards. Using the definition of the stress tensor in terms of perturbation pressure: $\sigma_{i j}=\tau_{i j}-p \delta_{i j}$, the governing equations become:

$$
\begin{array}{r}
-\sum_{j} \partial_{j} \sigma_{i j}=-\rho^{\prime} g k_{i}, \\
\sum_{i} \partial_{i} u_{i}=0 .
\end{array}
$$

Because $\sum_{i, j} n_{i} \sigma_{i j} n_{j}=\sum_{i, j} n_{i} \hat{\sigma}_{i j} n_{j}+\rho_{0} g\left(z_{0}-z\right)$, the normal component of (5b) changes to:

$$
\left.\sum_{i, j} n_{i} \sigma_{i j} n_{j}\right|_{z=z_{0}+\eta}=-\rho_{0} g \eta .
$$

Now the hydrostatic component has been removed, the vertical variation of $\sigma_{i j}$ near the free surface boundary has become significantly smaller. As a result, we can approximate the influence of topography in our model without actually changing the geometry, and apply boundary condition (7) at the reference level $z=z_{0}$. Since the local density $\rho$ may differ from the reference $\rho_{0}$, due to thermal and compositional heterogeneity, we also take into account the hydrostatic pressure variation associated with the perturbation density $\rho^{\prime}$, by using the full local density:

$$
\left.\sum_{i, j} n_{i} \sigma_{i j} n_{j}\right|_{z=z_{0}}=-\rho g \eta .
$$

Because this boundary condition is evaluated at a fixed level $z=z_{0}$, it can be easily implemented in a Eulerian model, without having to move the mesh. Note that in this case the normal $n_{i}$ is simply pointing upwards (or downwards for a bottom free surface), so that $\sum_{i, j} n_{i} \sigma_{i j} n_{j}$ simplifies to $\sigma_{z z}$ and the inner product $\sum_{i} n_{i} k_{i}$ in (5a) is simply a factor of +1 or -1 for top and bottom free surfaces, respectively.

The model equations with this boundary condition, the same as used in Zhong et al. (1996), form a linear system, whereas the zero normal stress condition (5b) applied at exactly 
$z=z_{0}+\eta$ leads to a non-linearity. However, the difference between the two formulations is only significant if the amplitude of the topography is of the same order as the horizontal lengthscales involved (as shown in Crameri et al., 2011).

To model the case where the zero normal stress condition is evaluated at exactly $z=z_{0}+\eta$, the computational mesh must be moved with the free surface, using, for example, ALE methods (Fullsack, 1995; Jimack and Wathen, 1991). We would like to emphasize that with such moving mesh methods, we can still subtract the reference hydrostatic pressure and use boundary condition (7). Note that in such cases, we have to use $\rho_{0}$ as opposed to $\rho$, since the hydrostatic pressure associated with the perturbation density is already taken into account by the change in volume of cells near the free surface. Using this form of the boundary condition however, the free surface method presented in the following sections remains applicable and offers the same second order accuracy and improved stability. The method is therefore equally applicable to ALE discretizations. In all results presented here however, a fixed Eulerian mesh is used as moving the mesh would have a negligible influence on the cases examined.

Finally, rather than assuming a zero external pressure, we may also consider the movement of a free surface into a region of different density $\rho_{\text {ext }}$. Examples include an ocean above Earth's crust and the higher density core below Earth's mantle. In such cases, we assume an ambient hydrostatic pressure, $\hat{p}_{\text {ext }}=-\rho_{\text {ext }} g z$, which modifies the normal stress boundary condition as follows:

$$
\left.\sum_{i, j} n_{i} \sigma_{i j} n_{j}\right|_{z=z_{0}}=-\Delta \rho g \eta
$$

where $\Delta \rho=\rho-\rho_{\text {ext }}$.

\subsection{Discrete method}

For the weak form of (6) we multiply the momentum equation and continuity equation by test functions $N$ and $M$ respectively, and integrate over the domain $\Omega$. Furthermore, we integrate by parts the deviatoric stress and velocity divergence terms, yielding surface integrals across the boundary $\Gamma$. The resulting equations are:

$$
\begin{gathered}
\int_{\Omega} \sum_{j}\left(\partial_{j} N\right) \tau_{i j}+N \partial_{i} p-\int_{\Gamma} N \sum_{j} n_{j} \tau_{i j}=-\int_{\Omega} N \rho^{\prime} g k_{i}, \\
\int_{\Omega} \sum_{i}\left(\partial_{i} M\right) u_{i}-\int_{\Gamma} \sum_{i} M n_{i} u_{i}=0 .
\end{gathered}
$$

On free slip and no slip boundaries, the normal velocity, $\sum_{i} n_{i} u_{i}$, is zero and the boundary integral drops out from the continuity equation. However, for free surface boundaries this integral remains. On these boundaries, we wish to weakly impose (9) and do so by substitution of this equation into the $\tau_{i j}$ boundary integral in the momentum equation:

$$
\begin{aligned}
\int_{\Omega} \sum_{j}\left(\partial_{j} N\right) \tau_{i j}+N \partial_{i} p-\int_{\Gamma_{\text {no } / \text { free slip }}} & N \sum_{j} n_{j} \tau_{i j} \\
& -\int_{\Gamma_{\text {free surface }}} N(p-\Delta \rho g \eta) n_{i}=-\int_{\Omega} N \rho^{\prime} g k_{i},
\end{aligned}
$$

$$
\int_{\Omega} \sum_{i}\left(\partial_{i} M\right) u_{i}-\int_{\Gamma_{\text {free surface }}} \sum_{i} M n_{i} u_{i}=0 .
$$


Explicit free surface

In this paper we assume a standard Continuous Galerkin finite element discretization of (11) as outlined in Davies et al. (2011), in which the discrete velocity and pressure solutions are written as linear combinations of a set of basis functions $N_{b}$ and $M_{a}$ respectively:

$$
u_{i}=\sum_{b} u_{b_{i}} N_{b} \text { and } p=\sum_{a} p_{a} M_{a} .
$$

This leads to a discrete linear system of equations:

$$
\left(\begin{array}{cc}
\mathrm{K} & \mathrm{G} \\
\mathrm{G}^{T} & 0
\end{array}\right)\left(\begin{array}{c}
\underline{u} \\
\underline{p}
\end{array}\right)=\left(\begin{array}{c}
f-\underline{h} \\
0
\end{array}\right),
$$

where $\underline{u}$ and $p$ are vectors of the coefficients $u_{b_{i}}$ and $p_{a}$. The matrices $\mathrm{K}$ and $\mathrm{G}$ and right-hand side vectors $\underline{f}$ and $\underline{h}$ are given by:

$$
\begin{aligned}
\mathrm{K}_{b_{i} c_{j}} & =\int_{\Omega}\left(\partial_{j} N_{b}\right) \mu\left(\partial_{i} N_{c}\right)+\sum_{k}\left(\partial_{k} N_{b}\right) \mu\left(\partial_{k} N_{c}\right) \delta_{i j} \\
\mathrm{G}_{b_{i} a} & =\int_{\Omega}\left(\partial_{i} M_{a}\right) N_{b}-\int_{\Gamma_{\text {free surface }}} M_{a} N_{b} n_{i}, \\
\underline{f}_{b_{i}} & =-\int_{\Omega} N_{b} \rho^{\prime} g k_{i}, \quad \underline{h}_{b_{i}}=\int_{\Gamma_{\text {free surface }}} N_{b} \Delta \rho g \eta n_{i} .
\end{aligned}
$$

Note that the pressure boundary integral in the momentum equation (11) and the normal velocity boundary integral in the continuity equation (both are integrals over the free surface) give rise to the same contribution to the discrete pressure gradient operator $G$ and the discrete divergence operator $G^{T}$, so that the system remains symmetric. The $\eta$ boundary integral in the momentum equation has been added as an additional term $-\underline{h}$ on the right-hand side. The boundary integrals for no-slip and free slip disappear through the application of strong Dirichlet boundary conditions for velocity and weakly imposing zero shear stress on the free slip boundaries (see Davies et al. (2011)).

The explicit free surface method tracks free surface elevation $\eta$ by a simple backward Euler time integration of the kinematic boundary condition, meaning that at the end of a timestep the free surface is updated using the latest velocity:

$$
\eta^{n+1}=\eta^{n}+\frac{\Delta t}{\sum_{i} n_{i} k_{i}} \sum_{i} u_{i}^{n} n_{i} .
$$

This new $\eta^{n+1}$ is used in the next timestep to compute the right-hand side term $\underline{h}$.

For consistency with the implicit method introduced in the next section, we define a rescaled free surface variable $\tilde{\eta}=\Delta \rho g \eta$, and discretize it by a linear combination of basis functions $Q_{d}$ :

$$
\tilde{\eta}=\sum_{d} \tilde{\eta}_{d} Q_{d}
$$

The functions $Q_{d}$ are finite element basis functions along the free surface only, associated with node $d$ in a surface mesh. For the results presented here we obtain these by restricting the 
pressure basis functions $M_{a}$ to the free surface. The equation that updates the free surface values after each timestep then follows from a standard Galerkin discretization:

$$
\sum_{e} \mathrm{~F}_{d e} \tilde{\eta}_{e}^{n+1}=\sum_{e} \mathrm{~F}_{d e} \tilde{\eta}_{e}^{n}+\Delta t \int_{\Gamma_{\text {free surface }}} Q_{a} \sum_{b, i} N_{b_{i}} u_{b_{i}} n_{i},
$$

where $\mathrm{F}$ is a free surface mass matrix:

$$
\mathrm{F}_{d e}=\int_{\Gamma_{\text {free surface }}} \frac{\sum_{i} n_{i} k_{i}}{\Delta \rho g} Q_{d} Q_{e} .
$$

It should be noted that this linear system, associated with matrix F can be easily solved, either with only a few iterations of a linear solver (used herein), or by lumping the matrix. Likewise, the actual free surface elevation $\eta$ can be recovered from $\tilde{\eta}$ in an equally trivial Galerkin projection. In all results presented here, we use a piecewise quadratic representation for velocity $u_{i}(\mathrm{P} 2)$ and a piecewise linear representation (P1) for pressure $p$ and free surface variable $\tilde{\eta}$. When the density is constant along the free surface (as is the case in all simulations examined herein), this implies that free surface elevation $\eta$ is also piecewise linear.

\section{Explicit method with non-linear iterations}

As will be shown in the following sections, the explicit method is only first order accurate. Improved accuracy can be obtained when multiple iterations are performed per timestep. In each iteration, the linear system is updated and solved. Such iterations are typically used when non-linearity is introduced to the system by, for example, coupling to a temperature advection-diffusion equation (see Davies et al., 2011). In this paper, such a method is used to update the free surface term $g$.

Suppose in the first iteration we have obtained a new free surface value $\tilde{\eta}^{*}$. In a subsequent iteration we may then use:

$$
\underline{h}_{b_{i}}=\int_{\Gamma_{\text {free surface }}} N_{b} \sum_{d} Q_{d}\left((1-\theta) \tilde{\eta}_{d}^{n}+\theta \tilde{\eta}_{d}^{*}\right) n_{i} .
$$

For $\theta=0$ we maintain the same fully explicit method. As will be shown in the following sections, setting $\theta=1 / 2$ leads to a scheme that is second order accurate in time.

\section{Implicit free surface}

For an implicit treatment of the free surface, the free surface values $\tilde{\eta}_{d}$ are stored in a vector $\underline{\tilde{\eta}}$ and solved for in conjunction with $\underline{u}$ and $\underline{p}$. The free surface integral term $\underline{h}$ is moved to the left-hand side of (13) and written in matrix form:

$$
\underline{h}=\mathrm{H} \underline{\tilde{\eta}}, \text { with } \mathrm{H}_{b_{i} d}=\int_{\Gamma_{\text {free surface }}} N_{b} Q_{d} n_{i} .
$$

The same matrix $\mathrm{H}$ can be used to write the discretized kinematic boundary condition (16) as:

$$
-\mathrm{H}^{T} \underline{u}+\mathrm{F} \frac{\tilde{\tilde{\eta}}^{n+1}-\tilde{\tilde{\eta}}^{n}}{\Delta t}=0
$$


This leads to the combined system:

$$
\left(\begin{array}{ccc}
\mathrm{K} & \mathrm{G} & \mathrm{H} \\
\mathrm{G}^{T} & 0 & 0 \\
\mathrm{H}^{T} & 0 & -\Delta t^{-1} \mathrm{~F}
\end{array}\right)\left(\begin{array}{c}
\underline{u} \\
\underline{p} \\
\underline{\tilde{\eta}}^{\underline{n+1}}
\end{array}\right)=\left(\begin{array}{c}
\frac{f}{0} \\
-\Delta t^{-1} \mathrm{~F} \underline{\tilde{\eta}}^{n}
\end{array}\right) .
$$

Comparing (13) and (21) we may recognize that the same symmetric saddle point structure has been preserved, meaning that the usual solution methods are available for this linear system (see May and Moresi, 2008, for an overview of preconditioned iterative methods for problems of this type). In effect, during the solution procedure, the vector of pressure degrees of freedom is extended with additional degrees of freedom along the free surface, and the pressure gradient matrix $G$ is similarly extended to $(G H)$. Note that, as the extra degrees of freedom are only defined on the free surface, this requires minimal additional computational cost to solve the system.

We follow the same pressure correction approach, known as Schur Complement Reduction, as outlined in Davies et al. (2011), but now based on the Schur complement:

$$
\mathrm{S}=\left(\begin{array}{l}
G^{T} \\
H^{T}
\end{array}\right) K^{-1}\left(\begin{array}{ll}
G & H
\end{array}\right)+\Delta t^{-1}\left(\begin{array}{cc}
0 & 0 \\
0 & \mathrm{~F}
\end{array}\right) .
$$

The same Schur complement matrix can be used in preconditioners for a fully coupled solution approach. Therefore the implicit free surface method may be implemented in a manner that is minimally invasive to solver strategies in existing geodynamical models.

\section{Crank Nicolson time integration}

In (21) only $\tilde{\eta}$ carries a superscript $n$ and $n+1$ to indicate the previous and current (to be solved for) time level. The solution of $\underline{u}$ and $\underline{p}$, on the other hand, does not depend on previous timesteps. To make (20) a second order time integration however, $\underline{u}$ needs to be interpreted as the velocity at $t^{n+1 / 2}$. We will therefore indicate the velocity solved for in the current timestep with $\underline{u}^{n+1 / 2}$. To ensure second order temporal convergence of the whole system, the pressure and free surface need to be evaluated at the same time level $t^{n+1 / 2}$ in the discrete momentum equation, the first row of (21). Therefore, we also interpret the pressure solved for in the current timestep to be at this time level and write $\underline{p}=\underline{p}^{n+1 / 2}$. The free surface elevation used in the momentum equation is weighted between between the beginning and end of timestep value: $(1-\theta) \tilde{\eta}^{n}+\theta \tilde{\eta}^{n+1}$. The system can now be rewritten, while maintaining the same symmetric form as before:

$$
\left(\begin{array}{ccc}
\mathrm{K} & \mathrm{G} & \theta \mathrm{H} \\
\mathrm{G}^{T} & 0 & 0 \\
\theta \mathrm{H}^{T} & 0 & -\theta \Delta t^{-1} \mathrm{~F}
\end{array}\right)\left(\begin{array}{c}
\underline{u}^{n+1 / 2} \\
\underline{p}^{n+1 / 2} \\
\underline{\tilde{\eta}}^{n+1}
\end{array}\right)=\left(\begin{array}{c}
\underline{f}-(1-\theta) \mathrm{H} \underline{\tilde{\eta}}^{n} \\
0 \\
-\theta \Delta t^{-1} \mathrm{~F} \underline{\tilde{\eta}}^{n}
\end{array}\right) .
$$

In the following section we will show that for $\theta=1 / 2$ (Crank Nicolson) we indeed obtain a second order accurate scheme, while for $\theta=1$ we obtain a scheme that is first order accurate in time.

\section{Test cases}

In this section we present several comparisons between the explicit and implicit free surface methods. We first demonstrate the order of accuracy of each method in space and 
time by evaluating the error in the free surface calculation against known analytical solutions (described in Appendix A). We also discuss the relative stability of the two methods for these cases. This is demonstrated further via a simulation of a lithospheric instability beneath a free surface, which with the explicit method at too large a timestep results in the so-called 'drunken sailor' instability.

\subsection{Analytical comparison}

We consider the idealized geometry, shown in Figure C.1, of a two-dimensional box, $0 \leq x \leq L,-D \leq z \leq 0$. Boundary conditions are defined on the top, bottom, left and right boundaries and an additional internal boundary surface is defined to allow internal fluxes to be specified in some cases. By varying the boundary and initial conditions, several test cases are produced which demonstrate advantages of the implicit method and deficiencies in its explicit counterpart.

Simulations are non-dimensionalized using the depth, $D$, as a lengthscale and a relaxation time as a timescale. $D$ and other physical parameters are defined in Table B.1. The relaxation timescale varies between simulations. For small scale features, where the wavelength of the topographic feature, $\lambda \ll D$, it is given by:

$$
\tau_{0}=\frac{2 k \mu_{0}}{|\Delta \rho| g},
$$

where $k=2 \pi / \lambda$ is the wavenumber. For very large scale features, where $\lambda$ is of the same order as $D$, the formulae for the exact relaxation timescales are presented in Appendix A. We will demonstrate that the relaxation time is an important indicator for the stability criterion of the explicit method, whereas the stability of the implicit method appears independent of the chosen timestep.

\section{Single free surface}

In the first case, we consider a single free surface, $\eta(x, t)$, at the top, $z=0$, of the domain. All other external boundaries have free slip conditions applied while no condition is applied at the internal boundary. The perturbation density, $\rho^{\prime}$, is zero throughout the simulation. However, an initial free surface is prescribed, given by:

$$
\eta(x, 0)=F_{0} \cos (k x),
$$

where $F_{0}=1 \times 10^{3} \mathrm{~m}$ is the initial free surface amplitude. The simulation is run for $\lambda=D / 4, D / 2, D, 2 D$ and $4 D$ using both the explicit and implicit free surface methods. The relaxation timescale (used for non-dimensionalization) for each wavelength considered is presented in Table B.2 along with the approximate relaxation timescale, $\tau_{0}(24)$, for comparison. In all cases, $L=\lambda$ and the mesh is made up of structured triangles with a vertical edge length, $\Delta z$, of $D / 80$ and a horizontal edge length, $\Delta x$, of $L / 80$.

For both the implicit and explicit cases nine different timestep sizes are considered. The explicit method is not stable beyond a timestep $\Delta t$ of the relaxation time $\tau$, so simulations are performed between limits of $\Delta t=\tau$ and $\Delta t=\tau / 256$. All simulations attempted beyond a timestep of $\tau$ rapidly became unstably unbounded. The implicit case is not limited by timestep size, which we vary from $32 \tau$ to $\tau / 8$. 
At each timestep of the simulation the error between the free surface elevation, $\eta$, and the analytical solution, $\eta^{*}$ (see Appendix A), is evaluated in a surface $L 2$ norm on the top domain boundary ( $\eta^{*}$ is also treated as a piecewise linear function for this calculation). At the end of the simulation, these spatial errors are squared, weighted by the timestep, $\Delta t$, and summed into a discrete temporal $L 2$ norm, which is then used to test the temporal order of convergence of both methods. In the implicit case this temporal norm is taken over 64 relaxation times (so the largest timestep calculation performs only two timesteps). Due to the small timesteps required, and the resulting long simulation times, the temporal error was evaluated over 4 relaxation times for the explicit method (four timesteps in the largest $\Delta t$ case).

The results using a single iteration per timestep of the explicit method and $\theta=1 / 2$ (see equation (23)) for the implicit method are presented in Figure C.2(a). These demonstrate that the implicit method is temporally second order accurate compared to the first order accuracy of the explicit method at a wide range of wavelengths. At the limit of stability for the explicit method, $\Delta t=\tau$, the same error can be achieved with the implicit method with a timestep twice as large. At the other end of the timestep range considered, the lowest errors achieved by the explicit method can be reproduced with the implicit method with a timestep 50 times larger.

The order of convergence of the explicit method may be improved to match the implicit method at the cost of an extra iteration per timestep if $\theta$ (see equation (18)) is set to $1 / 2$, as shown in Figure C.2(b). However, the absolute error of the implicit, $\theta=1 / 2$, case is still lower and the iterated explicit method remains limited to $\Delta t \leq \tau$. The accuracy of the implicit method may also be reduced by selecting $\theta=1$ (see equation (23)), which results in it becoming almost indistinguishable from the explicit method (see Figure C.2(b)) at timesteps lower than the relaxation time.

Above a timestep of approximately three times the relaxation time the implicit, $\theta=1 / 2$, method, though still stable and converging to the correct steady state, becomes oscillatory. This can be seen in Figure C.2(c), where the free surface elevation, $\eta$ is shown at $x=0$ over 20 relaxation times. At this timestep, $\Delta t=4 \tau$, the method is effectively 'stepping over' the physics of the free surface. The oscillations may be damped out by selecting $\theta=1$. In fact, at this timestep, the $\theta=1$ case appears more accurate than with $\theta=1 / 2$ (see Figure C.2(b)), though this roll-over may be an artefact of the discrete temporal $L 2$ norm used. Once the free surface evolution is resolved by the $\theta=1 / 2$ implicit case it is clearly more accurate, being almost indistinguishable from the analytical solution in Figure C.2(c) at $\Delta t=\tau$.

\section{Two free surfaces}

In the second analytical comparison we modify the single free surface set-up to include a free surface, $\zeta(x, t)$, at the bottom of the domain, $z=-D$, representing the core-mantle boundary. This is initialized to:

$$
\zeta(x, 0)=G_{0} \cos (k x),
$$

where $G_{0}=1 \times 10^{3} \mathrm{~m}$ is the initial free surface amplitude. The simulation is run for $\lambda=D / 2, D, 2 D$ and $4 D$ using both the explicit and implicit methods. The relaxation timescales for each wavelength considered are presented in Table B.3 alongside the approximate relaxation timescale, $\tau_{0}(24)$, for comparison. The minimum of the timescales, $\tau_{-}$, is used for non-dimensionalization. 
Bounding the domain at the top and bottom by two free surfaces introduces a null space into the $z$ component of velocity, $w$. We remove this by pinning $w$ to zero at the stationary point $(x, z)=(L / 4,-D / 2)$. All other details of the simulation set-up remain the same as in the single free surface case.

Temporal convergence results for the top free surface $\eta$ are presented in Figure C.3 (results for the lower free surface $\zeta$ are identical). Again the errors in the explicit free surface method are evaluated over four minimum relaxation timescales. However, at the larger timesteps considered, simulations were also performed over a time period of $t=64 \tau_{-}$. These were found to be unstable when the timestep exceeded $\tau_{-} / 8$ or $\tau_{-} / 16$, depending on the wavelength. Simulations above these timesteps are excluded from the graph.

For the single free surface case, the largest stable timestep for the explicit method (as presented in Figure C.2(a)) was found to be $\Delta t=\tau$ when run for the same, longer time, $t=64 \tau$. It therefore appears that having two free surfaces makes the explicit method more unstable. It may also be the case that the single free surface would exhibit instability at $\Delta t \leq \tau$ if the simulations were run for even longer time periods. In the two free surface case, the instability in the explicit method for $\Delta t \gtrsim \tau_{-} / 16$ means that no explicit method with two iterations per timestep was stable in the error window being considered. Hence we only present results for the explicit method with the same number of solves per timestep as the implicit method, i.e. one.

As with the single free surface case, the implicit method for two free surface is seen to be stable across all timesteps considered and result in second order convergence. The apparently lower limit of stability for the explicit method means that, at its highest error, a timestep approximately 10 times larger may be taken with the implicit method, while, at its lowest error, the ratio is still about 50 across a range of wavelengths.

\section{Two free surfaces with density anomaly}

The final analytical comparison was proposed by Zhong et al. (1996). This keeps the top and bottom free surfaces from the previous case but with initial amplitudes of $F_{0}=0$ and $G_{0}=0$ respectively. In addition, a buoyancy anomaly is introduced such that:

$$
\rho^{\prime} g=-\rho_{0} \alpha \Delta T g
$$

where $g, \rho_{0}$ and $\alpha$ are the gravity magnitude, reference density and thermal expansivity respectively (see Table B.1) and $\Delta T$ is a temperature anomaly:

$$
\Delta T=Q \cos (k x) \delta(z+d),
$$

where $Q$ is a scaling parameter (see Table B.1) and $\delta(z+d)$ is a Dirac delta function located at depth, $d$. Once integrated with respect to $z$ we introduce this buoyancy as a weakly imposed vertical momentum flux over the internal surface indicated in Figure C.1. The vertical velocity is also pinned at the stationary point $(x, z)=(L / 4,-d)$ to remove the null space.

The buoyancy anomaly establishes convection within the domain and drives the free surfaces towards a non-zero amplitude steady state. Two anomaly depths, $d=D / 2$ and $d=D / 4$, are considered at a wavelength of $\lambda=D$ between timesteps of $32 \tau_{-}$and $\tau_{-} / 8$ in the implicit case and $\tau_{-}$and $\tau_{-} / 128$ in the explicit case. As was the case without a buoyancy anomaly, simulations with $\Delta t \gtrsim \tau_{-} / 16$ were found to be unstable with the explicit method. 
The relaxation timescales are identical to the case with no buoyancy and are given in Table B.3 for $\lambda=D$.

Temporal convergence results are presented in Figure C.4(a) and again demonstrate second order convergence for the implicit method versus first order for the explicit case with the same number of iterations per timestep (one). Solution accuracy is illustrated in Figure C.4(b) where $\eta$ and $\zeta$ are plotted at $x=0$ for five minimum relaxation timescales. The solution for $\Delta t=\tau_{-} / 4$ is indistinguishable from the analytical solution (see Appendix A) and yet was run with a timestep four times larger than possible with the explicit method and achieves an error over five times lower (see Figure C.4(a)).

All results presented in Figure C.4 were performed on a structured triangular mesh with a vertical edge length, $\Delta z$, of $D / 160$ and a horizontal edge length, $\Delta x$, of $L / 160$. The increased resolution relative to the previous cases was found to be necessary as erroneous temporal convergence occurred at lower resolutions for both methods (see Figure C.5(a)). This is due to the high solution gradients induced by the buoyancy anomaly delta function within the domain. As illustrated in Figure C.5(b), for the $d=D / 2$ case, the pressure solution is highly discontinuous and, at lower resolutions, e.g. $\Delta x / L=\Delta z / D=1 / 20$, is poorly represented causing, with decreasing timesteps, convergence to an incorrect solution.

Finally, we test the spatial convergence of both methods by timestepping to a steady state, within a tolerance of $10^{-10}$, and evaluating the free surface error in an $L 2$ norm over the top and bottom of the domain. Here the implicit method takes timesteps of $100 \tau_{-}$while the explicit method is limited by stability to $\Delta t=\tau_{-} / 16$. As the free surface in both cases is piecewise linear in space, both are seen to achieve second order spatial accuracy, as shown in Figure C.5(c) for $\eta$ with $d=D / 2$.

All three analytical cases demonstrate that, when configured with $\theta=1 / 2$, the implicit method achieves a higher order of convergence than the explicit method with a single solve per timestep. While the explicit method's accuracy may be improved by increasing the number of iterations, and doubling the solver cost, per timestep, its stability is severely limited by the timestep size, which is not the case with the implicit method. We proceed to investigate the stability properties of both methods further with a geophysically motivated example, the case of a lithospheric instability beneath a free surface.

\subsection{Rayleigh-Taylor instability: 'drunken sailor'}

In this section we simulate the Rayleigh-Taylor instability proposed by Kaus et al. (2010). A square two-dimensional domain, $-2.5 \times 10^{5} \mathrm{~m} \leq x \leq 2.5 \times 10^{5} \mathrm{~m},-5 \times 10^{5} \mathrm{~m} \leq z \leq 0 \mathrm{~m}$, is considered with free slip velocity boundary conditions at the sides, a no slip boundary condition at the base and a free surface boundary condition at the top. Inside the domain, a dense material of higher viscosity $\left(\rho_{1}=3300 \mathrm{~kg} \mathrm{~m}^{-3}, \mu_{1}=1 \times 10^{21} \mathrm{Pas}\right)$ is initially above a material of lower density and viscosity $\left(\rho_{1}=3200 \mathrm{~kg} \mathrm{~m}^{-3}, \mu_{2}=1 \times 10^{20} \mathrm{Pas}\right)$. The materials are separated by a sharp material boundary, which is initially at a depth of $1 \times 10^{5} \mathrm{~m}$ with a sinusoidal perturbation, $5 \times 10^{3} \mathrm{~m}$ in amplitude:

$$
z=-1 \times 10^{5} \mathrm{~m}+5 \times 10^{3} \mathrm{~m} \cos \left(\frac{2 \pi x}{5 \times 10^{5} \mathrm{~m}}\right) .
$$

Over time this unstable stratification produces two dense drips of material that flow down the sides of the domain. The free surface, initialized with no topography, responds to the internal deformation by warping down above the drips and up in the center of the domain. 
The locations of the different materials are indicated by a volume fraction, $\phi$, which is equal to one in the dense upper material and zero in the lower material. The density and viscosity are then given by:

$$
\begin{aligned}
& \rho=\rho_{1} \phi+\rho_{2}(1-\phi), \\
& \mu=\mu_{1} \phi+\mu_{2}(1-\phi) .
\end{aligned}
$$

Taking the hydrostatic reference density as that of the dense upper material bordering the free surface, $\rho_{0}=\rho_{1}$, the perturbation density is given by:

$$
\rho^{\prime}=\left(\rho_{2}-\rho_{1}\right)(1-\phi) \text {. }
$$

The volume fraction, $\phi$, is advected using a linear advection equation:

$$
\frac{\partial \phi}{\partial t}+\sum_{i} u_{i} \partial_{i} \phi=0,
$$

which is discretized in space on a dual mesh using a finite volume flux-limited scheme (HyperC, Leonard, 1991; Wilson, 2009). The control volumes of the dual mesh are defined around the $\mathrm{P} 2$ nodes of the finite element mesh. Advection is treated explicitly in time and subcycled to ensure a maximum Courant number, $C$, of $1 / 4$.

This case is used to examine the relative stabilities of the explicit and implicit free surface methods over a time period of $t=6 \mathrm{Myr}$. In the explicit case the timestep size, $\Delta t$, is defined at the start of the simulation and held constant for its duration. In the implicit case a target advective Courant number, $C$, is set on the dual mesh. The timestep is then automatically adjusted, based on the velocity of the previous timestep, $u_{i}^{n}$, in an attempt to ensure that the target Courant number is maintained. This process is restricted to ensure that large jumps in the timestep do not occur at the start of the simulation when the velocity is initially zero. Iterations between the velocity/pressure/free surface and volume fraction solution steps are used to converge the non-linearity in the coupled system. The domain is discretized using a structured triangular mesh with equal $5 \times 10^{3} \mathrm{~m}$ vertical and horizontal edge lengths.

Simulations employing an explicit free surface treatment with one non-linear iteration were found to be stable at $\Delta t \leq 4000$ years. At these timesteps the topography amplitude increases gradually, but decays towards the end of the simulation, as high density material reaches the base of the domain and the velocity decreases again (see Figures C.6(a) and C.8). However, at larger timesteps a sloshing instability occurs, where the velocity field oscillates between consecutive timesteps, eventually becoming physically unbounded (see Figure C.7). These results are consistent with those of Kaus et al. (2010). When two non-linear iterations are used, the explicit method is unstable with $\Delta t>2000$ years. Since we would hope that the solution would converge with non-linear iterations, the explicit free surface treatment is considered unstable at $\Delta t>2000$ years, which is substantially less than the estimated free surface relaxation time of approximately 25000 years for this problem (based on equation (24)).

The implicit free surface treatment, by contrast, remains stable at significantly larger timesteps and with an increasing number of non-linear iterations (five are used in the implicit results discussed below). Although the timestep size varies substantially throughout the simulation (see Figure C.8(a)), it is consistently at least an order of magnitude greater than 
that of the stable explicit case ( $\Delta t=2000$ years), varying between $\Delta t \approx 25000$ years and $\Delta t \approx 600000$ years (targeting a Courant number, $C$, of 5 ). Nonetheless, solution accuracy is maintained at these larger timesteps, as demonstrated by a comparison of the material interface and free surface evolution to the stable explicit simulation (see Figure C.8(b, c)). It should be noted that the implicit free surface simulation evolves at similar timesteps to a simulation with a free slip top boundary condition targeting the same Courant number (Figure C.8(a)). Indeed, the computational requirements of both cases are very similar, with the implicit free surface simulation taking only a factor of 1.04 greater wall-time than the free slip case. On the other hand, the explicit free surface simulation, running at timesteps that are a fraction of those of the free slip case and with fewer non-linear iterations, took a factor of 21.96 greater wall-time.

\section{Conclusions}

We have presented a new algorithm for simulating a free surface in geodynamical models. The method couples the equation for the evolution of free surface elevation to the Stokes system of velocity and pressure, using implicit time integration. It is straightforward to implement, does not complicate the solution procedure and requires similar computation per timestep as a standard free slip case. The implicit time integration allows simulations to evolve at much larger time steps when compared to previous explicit free surface methods. Moreover, the method is second order accurate in time, meaning that the same level of accuracy is achieved even at these larger timesteps. The method therefore substantially reduces the computational cost of free surface simulations.

We have demonstrated these benefits by comparing model predictions to a number of analytical cases. These clearly show that although accurate, the explicit method is bound by timesteps that can be an order of magnitude smaller than the relaxation time for the problem under examination. Such small timestep constraints would render free surface simulations prohibitively expensive for large scale geodynamical models. Conversely, the new implicit method remains stable at much larger timesteps, while producing the same accurate solutions. We have also demonstrated that for a well known Rayleigh-Taylor benchmark case, the drunken sailor instability, reported in Kaus et al. (2010) and reproduced here with the explicit method, does not occur with the implicit free surface treatment. Again for this case, we show that the implicit method remains accurate at larger timesteps, leading to a dramatic reduction in computational cost when compared to the explicit case.

It should be noted that although the implicit method remains stable at timesteps that are orders of magnitude larger than the relaxation time of the problem under examination, the relaxation of these large scale features would clearly not be resolved with such timesteps. In such cases, the large scale response of the free surface to changes in the interior would be immediate, leading to similar predictions of free surface elevation as those computed from a free slip calculation. However the relaxation time for smaller scale features in the topography may be much larger. Accurately resolving the response time of such features is only possible with a true free surface and it is therefore essential when these smaller scale features are embedded in a large scale model to apply a free surface method that remains stable in such conditions. 


\section{Acknowledgements}

CRW was funded by NSF grant OCE0841079, DRD by a NERC post-doctoral Research Fellowship (NE/H015329/1). The authors would like to acknowledge support from the Applied Modelling and Computation Group (AMCG) at Imperial College London and thank Marc Spiegelman for many interesting discussions and encouragement.

\section{Appendix A. Analytical solutions}

In this appendix we work out the analytical solutions for the benchmarks of Section 3.1. We follow the approach of Zhong et al. (1996) that combines approaches of Solomon et al. (1982) and Parsons and Daly (1983). Similar analytical solutions are also provided in Ramberg (1967).

We consider a domain in 2 dimensions, $0 \leq x \leq L$ and $-D \leq z \leq 0$, where $D$ represents the depth of Earth's mantle. For a divergence free velocity field $(u, w)$, we can find a stream function $\psi$ such that:

$$
u=-\frac{\partial \psi}{\partial z}, \quad w=\frac{\partial \psi}{\partial x}
$$

After substitution of this into the momentum equation of (1) and taking its curl, we obtain:

$$
\frac{\partial^{4} \psi}{\partial x^{4}}+2 \frac{\partial^{4} \psi}{\partial x^{2} \partial z^{2}}+\frac{\partial^{4} \psi}{\partial z^{4}}=-\frac{\partial f}{\partial x}
$$

where $f=-\rho^{\prime} g$ is the buoyancy term in the vertical momentum equation. A generic solution for the homogeneous case $f=0$ that satisfies reflective boundary conditions at $x=0$ and $x=L$ is given by:

$$
\psi(x, z, t)=\sin (k x)(A(t) \sinh (k z)+B(t) \cosh (k z)+C(t) z \sinh (k z)+E(t) z \cosh (k z)),
$$

with $k=2 \pi / \lambda$ where $\lambda$ is an integer fraction of $L$.

\section{Single free surface}

In this case we consider a free surface condition at the top of the domain, $z=0$, a free slip condition at the bottom, $z=-D$, and no buoyancy forcing $(f=0)$. An expression for the free surface that conforms to solution (A.3) is given by:

$$
\eta^{*}(x, t)=F(t) \cos (k x) .
$$

The free slip condition (4) provides us with 2 equations, the no normal flow and the zero shear stress condition. The free surface condition (5) at the top provides 3 equations because in addition to the kinematic boundary condition and zero shear stress, it also enforces a no normal stress condition. More specifically, we consider the no normal stress condition (8) evaluated at $z=0$. To evaluate this boundary condition however, we need to know what the pressure is. In order to avoid this problem, we take the $x$-derivative of the boundary 
condition; the $x$-derivative of the pressure can then be eliminated by substitution of the $x$-component of the momentum equation:

$$
\begin{aligned}
\left.\frac{\partial \sigma_{z z}}{\partial x}\right|_{z=0}=\frac{\partial \tau_{z z}}{\partial x}-\left.\frac{\partial p}{\partial x}\right|_{z=0} & =-\left.g \rho \frac{\partial \eta^{*}}{\partial x}\right|_{z=0}, \\
\frac{\partial \tau_{z z}}{\partial x}-\frac{\partial \tau_{x x}}{\partial x}-\left.\frac{\partial \tau_{z x}}{\partial z}\right|_{z=0} & =-\left.g \rho \frac{\partial \eta^{*}}{\partial x}\right|_{z=0}, \\
-2 A(t) k^{3} \mu \sin (k x) & =\rho g F(t) k \sin (k x) .
\end{aligned}
$$

These five equations form a linear system for the variables $A, B, C, E$ and $F$ which we can solve for. In particular, the kinematic boundary condition introduces a time derivative of $F$ so that we obtain the simple ODE:

$$
F(t)=-\tau \frac{d F(t)}{d t}
$$

where $\tau$ is the relaxation time:

$$
\tau=\frac{D k+\sinh (D k) \cosh (D k)}{\sinh ^{2}(D k)} \tau_{0}, \quad \tau_{0}=\frac{2 k \mu}{\rho g} .
$$

Using the initial condition $F(0)=F_{0}$, the solution is given by:

$$
\begin{array}{r}
F(t)=F_{0} e^{-t / \tau}, \\
A(t)=-\frac{F_{0}}{k \tau_{0}} e^{-t / \tau}, \quad B(t)=-\frac{F_{0}}{k \tau} e^{-t / \tau}, \\
C(t)=\frac{F_{0}}{\tau} e^{-t / \tau}, \quad E(t)=\frac{F_{0}}{\tau \tanh (D k)} e^{-t / \tau} .
\end{array}
$$

\section{Two free surfaces}

In this case we also consider a free surface at the bottom of the domain, given by $z=$ $-D+\zeta^{*}:$

$$
\zeta^{*}(x, t)=G(t) \cos (k x) .
$$

In addition to replacing the no-normal flow condition at the bottom with the kinematic boundary condition, this introduces a sixth equation in the form of a no-normal-stress condition, which we express in the form of (9) using $\Delta \rho=\rho_{\zeta}-\rho_{\text {ext }}$ where $\rho_{\text {ext }}$ represents the approximate density in the outer core. Note that this implies $\Delta \rho<0$. Here, we use $\rho_{\eta}$ and $\rho_{\zeta}$ for the densities at (the inside of) the top and bottom boundary respectively.

Again, the boundary condition forms a linear system of equations, which we can solve for $A, B, C, E, F$ and $G$. The kinematic boundaries have introduced time derivatives of both $F$ and $G$ so that we obtain a 2 variable ODE:

$$
\left(\begin{array}{l}
F \\
G
\end{array}\right)=-\left(\begin{array}{cc}
\tau_{\eta} & -\gamma \tau_{\eta} \\
-\gamma \tau_{\zeta} & \tau_{\zeta}
\end{array}\right)\left(\begin{array}{l}
\frac{\partial F}{\partial t} \\
\frac{\partial G}{\partial t}
\end{array}\right),
$$


where we use the following timescales $\tau_{\eta}$ and $\tau_{\zeta}$ associated with the free surface at the top and bottom respectively, and a factor $\gamma$ :

$$
\begin{aligned}
\tau_{\eta}=\frac{D k+\sinh (D k) \cosh (D k)}{\sinh ^{2}(D k)} \tau_{\eta, 0}, & \tau_{\eta, 0}=\frac{2 k \mu}{\rho_{\eta} g} \\
\tau_{\zeta}=\frac{D k+\sinh (D k) \cosh (D k)}{\sinh ^{2}(D k)} \tau_{\zeta, 0}, & \tau_{\zeta, 0}=\frac{2 k \mu}{-\Delta \rho g}, \\
\gamma & =\frac{D k \cosh (D k)+\sinh (D k)}{D k+\sinh (D k) \cosh (D k)} .
\end{aligned}
$$

The eigenvalues of the ODE matrix gives two relaxation times:

$$
\tau_{ \pm}=\frac{1}{2}\left(\tau_{\eta}+\tau_{\zeta}\right) \pm \frac{1}{2} \sqrt{\left(\tau_{\eta}+\tau_{\zeta}\right)^{2}-4\left(1-\gamma^{2}\right) \tau_{\eta} \tau_{\zeta}}
$$

Note that $0 \leq \gamma \leq 1$ and that for $\lambda \ll D, \gamma$ rapidly goes to zero, so that, as expected, the top and bottom free surface time scales become independent.

For initial conditions $F(0)=F_{0}$ and $G(0)=G_{0}$, we obtain:

$$
\begin{array}{r}
F(t)=\frac{F_{0}\left(\tau_{\eta}-\tau_{-}\right)-\gamma G_{0} \tau_{\eta}}{\tau_{+}-\tau_{-}} e^{-t / \tau_{+}}-\frac{F_{0}\left(\tau_{\eta}-\tau_{+}\right)-\gamma G_{0} \tau_{\eta}}{\tau_{+}-\tau_{-}} e^{-t / \tau_{-}}, \\
G(t)=\frac{G_{0}\left(\tau_{\zeta}-\tau_{-}\right)-\gamma F_{0} \tau_{\zeta}}{\tau_{+}-\tau_{-}} e^{-t / \tau_{+}}-\frac{G_{0}\left(\tau_{\zeta}-\tau_{+}\right)-\gamma F_{0} \tau_{\zeta}}{\tau_{+}-\tau_{-}} e^{-t / \tau_{-}}, \\
A(t)=-\frac{F(t)}{k \tau_{\eta, 0}}, \quad B(t)=k^{-1} \frac{\partial F}{\partial t}, \quad C(t)=-\frac{\partial F}{\partial t}, \\
E(t)=\frac{\frac{\partial G}{\partial t}-\cosh (D k) \frac{\partial F}{\partial t}}{\sinh (D k)} .
\end{array}
$$

Two free surfaces with density anomaly

This is the benchmark given in Zhong et al. (1996), where in addition to a bottom and top free surface, we now also consider a density anomaly $\rho^{\prime}=-\alpha \rho_{0} Q \cos (k x) \delta(z+d)$ at a depth $z=-d$ which gives a right-hand side term in the momentum equation:

$$
f=\rho_{0} g \alpha Q \cos (k x) \delta(z+d),
$$

where $Q$ represents the amplitude scaling of the temperature anomaly. The solution for this case is derived, following Zhong et al. (1996), by splitting the generic solution in two parts:

$$
\psi(x, z, t)=\left\{\begin{array}{cl}
\sin (k x)\left(A_{1}(t) \sinh (k z)+B_{1}(t) \cosh (k z)+\right. & \text { if }-d<z<0, \\
\left.C_{1}(t) z \sinh (k z)+E_{1}(t) z \cosh (k z)\right) & \\
\sin (k x)\left(A_{2}(t) \sinh (k z)+B_{2}(t) \cosh (k z)+\right. & \text { if }-D<z<-d . \\
\left.C_{2}(t) z \sinh (k z)+E_{2}(t) z \cosh (k z)\right) &
\end{array}\right.
$$

In addition to the six equations associated with the top and bottom free surface boundary condition, we need four further equations to solve for $A_{1}, B_{1}, C_{1}, E_{1}, A_{2}, B_{2}, C_{2}, E_{2}, F$ and $G$. 
(A.1), and continuity of shear stress:

$$
\tau_{x z}=\mu \frac{\partial u}{\partial z}+\mu \frac{\partial w}{\partial x}=\mu\left(\frac{\partial^{2}}{\partial x^{2}}-\frac{\partial^{2}}{\partial z^{2}}\right) \psi=-\mu\left(k^{2}+\frac{\partial^{2}}{\partial z^{2}}\right) \psi .
$$

Continuity is therefore enforced through:

$$
\lim _{z \uparrow-d} \psi=\lim _{z \downarrow-d} \psi, \quad \lim _{z \uparrow-d} \frac{\partial \psi}{\partial z}=\lim _{z \downarrow-d} \frac{\partial \psi}{\partial z}, \quad \lim _{z \uparrow-d} \frac{\partial^{2} \psi}{\partial z^{2}}=\lim _{z \downarrow-d} \frac{\partial^{2} \psi}{\partial z^{2}} .
$$

Finally, a jump condition for the third derivative of $\psi$ can be derived from:

$$
\begin{aligned}
\lim _{\epsilon \rightarrow 0} \int_{-d-\epsilon}^{-d+\epsilon} \frac{\partial^{4} \psi}{\partial x^{4}}+2 \frac{\partial^{4} \psi}{\partial x^{2} \partial z^{2}}+\frac{\partial^{4} \psi}{\partial z^{4}} & =\lim _{\epsilon \rightarrow 0} \int_{-d-\epsilon}^{-d+\epsilon}-\frac{\partial f}{\partial x} \\
\lim _{z \downarrow-d} \frac{\partial^{3} \psi}{\partial z^{3}}-\lim _{z \uparrow-d} \frac{\partial^{3} \psi}{\partial z^{3}} & =\rho_{0} \alpha g Q k \sin (k x) .
\end{aligned}
$$

The solution of the ten-equation linear system again gives rise to an ODE in $F$ and $G$.

$$
\left(\begin{array}{c}
F \\
G
\end{array}\right)=-\left(\begin{array}{cc}
\tau_{\eta} & -\gamma \tau_{\eta} \\
-\gamma \tau_{\zeta} & \tau_{\zeta}
\end{array}\right)\left(\begin{array}{c}
\frac{\partial F}{\partial t} \\
\frac{\partial G}{\partial t}
\end{array}\right)+\left(\begin{array}{c}
M \\
N
\end{array}\right)
$$

where the inhomogeneous part is given by:

$$
\begin{aligned}
& M=\frac{\alpha \rho_{0} Q}{\rho_{\eta}} \frac{-k D \sinh (k d)+k d \cosh (k(D-d)) \sinh (k D)+\sinh (k(D-d)) \sinh (k D)}{\sinh ^{2}(k D)} \\
& N=\frac{\alpha \rho_{0} Q}{-\Delta \rho} \frac{k D \sinh (k d) \cosh (k D)-k d \cosh (k d) \sinh (k D)+\sinh (k d) \sinh (k D)}{\sinh ^{2}(k D)} .
\end{aligned}
$$

When reaching a steady state, the time derivatives vanish and therefore the steady solution is given by $F(t \rightarrow \infty)=M$ and $G(t \rightarrow \infty)=N$. Note that the homogeneous part of the ODE is the same as in the previous case with no forcing. This means the solution for $F$ and $G$ is the same when considered as a deviation from the $t \rightarrow \infty$ steady state limit:

$$
\begin{aligned}
& F(t)=\frac{\left(F_{0}-M\right)\left(\tau_{\eta}-\tau_{-}\right)-\gamma\left(G_{0}-N\right) \tau_{\eta}}{\tau_{+}-\tau_{-}} e^{-t / \tau_{+}}-\frac{\left(F_{0}-M\right)\left(\tau_{\eta}-\tau_{+}\right)-\gamma\left(G_{0}-N\right) \tau_{\eta}}{\tau_{+}-\tau_{-}} e^{-t / \tau_{-}}+M, \\
& \tau_{+}-\tau_{-}
\end{aligned}
$$


The coefficients for the solution of $\psi$ are given by:

$$
\begin{aligned}
& A_{1}(t)=-\frac{F(t)}{k \tau_{\eta, 0}}, \quad B_{1}(t)=k^{-1} \frac{\partial F}{\partial t}, \quad C_{1}(t)=-\frac{\partial F}{\partial t} \\
& E_{1}(t)=\frac{\frac{\partial G}{\partial t}-\cosh (D k) \frac{\partial F}{\partial t}+\xi \sinh (k(D-d))}{\sinh (D k)}, \\
& A_{2}(t)=\frac{-F(t)}{k \tau_{\eta, 0}}+k^{-1} \xi(\cosh (k d)-k d \sinh (k d)) \\
& B_{2}(t)=k^{-1} \frac{\partial F}{\partial t}+k^{-1} \xi(\sinh (k d)-k d \cosh (k d)) \\
& C_{2}(t)=-\frac{\partial F}{\partial t}-\xi \sinh (k d), \\
& E_{2}(t)=\frac{\frac{\partial G}{\partial t}-\cosh (D k) \frac{\partial F}{\partial t}-\xi \sinh (k d) \cosh (k D)}{\sinh (D k)}
\end{aligned}
$$

where $\xi$ is given by:

$$
\xi=\frac{\alpha Q \rho_{0} g}{2 k \mu} .
$$

\section{References}

C. Beaumont, P. Kamp, J. Hamilton, and P. Fullsack. The continental collision zone, South Island, New Zealand: comparison of geodynamical models and observations. J. Geophys. Res., 101:3333-3359, 1996.

C. Beaumont, R. A. Jamieson, M. H. Nguyen, and B. Lee. Himalayan tectonics explained by extrusion of a low-viscosity crustal channel coupled to focused surface denudation. Nature, 414:738-742, 2001. doi: 10.1038/414738a.

J. Braun. Recent advances and current problems in modelling surface processes and their interactions with tectonics and crustal deformation. In: Buiter, S. J. H., and Schreurs G. (Eds.), analog and numerical modelling of crustal-scale processes. Spec. Pub. Geol. Soc. London, pages 307-325, 2006.

J. Braun. The many surface expressions of mantle dynamics. Nature Geoscience, 3:826-833, 2010. doi: 10.1038/ngeo1020.

H.-P. Bunge, M. A. Richards, and J. R. Baumgardner. Mantle circulation models with sequential data-assimilation: Inferring present-day mantle structure from plate motion histories. Phil. Trans. R. Soc. London, Set. A, 360:2545-2567, 2002. doi: 10.1098/rsta.2002.1080.

F. Crameri, H. Schmeling, G. J. Golabek, T. Duretz, R. Orendt, S. Buiter, D. A. May, B. J. P. Kaus, T. V. Gerya, and P. J. Tackley. A benchmark comparison of numerical surface topography calculations in geodynamic modelling. Geophys. J. Int., In press., 2011.

D. R. Davies and J. H. Davies. Thermally-driven mantle plumes reconcile multiple hot-spot observations. Earth and Planetary Science Letters, 278:50-54, 2009. doi: 10.1016/j.epsl.2008.11.027. 
D. R. Davies, C. R. Wilson, and S. C. Kramer. Fluidity: A fully unstructured anisotropic adaptive mesh computational modeling framework for geodynamics. Geochem. Geophys. Geosys., 120:Q06001, 2011. doi: 10.1029/2011GC003551.

L. Fuchs, H. Schmeling, and H. Koyi. Numerical models of salt diapir formation by downbuilding: the role of sedimentation rate, viscosity contrast, initial amplitude and wavelength. Geophys. J. Int., 186:390-400, 2011. doi: 10.1111/j.1365-246X.2011.05058.x.

P. Fullsack. An arbitrary Lagrangian-Eulerian formulation for creeping flows and its application in tectonic models. Geophys. J. Int., 120:1-23, 1995.

M. Gurnis, C. Eloy, and S. Zhong. Free-surface formulation of mantle convection: II. implication for subduction-zone observables. Geophys. J. Int, 127:719-727, 1996.

B. H. Hager, R. W. Clayton, M. A. Richards, R. P. Comer, and A. M. Dziewonski. Lower mantle heterogeneity, dynamic topography and the geoid. Nature, 313:541-545, 1985. doi: $10.1038 / 313541 \mathrm{a} 0$.

F. H. Harlow and J. E. Welch. Numerical calculation of time-dependent viscous incompressible flow of fluid with free surface. Phys. Fluids, 8:2182-2189, 1965.

G. Iaffaldano, H.-P. Bunge, and T. Dixon. Feedback between mountain belt growth and plate convergence. Geology, 34:893-896, 2006. doi: 10.1130/G22661.1.

G. Iaffaldano, L. Husson, and H.-P. Bunge. Monsoon speeds up Indian plate motion. Earth Planet. Sci. Lett., 304:503-510, 2011. doi: 10.1016/j.epsl.2011.02.026.

P. K. Jimack and A. J. Wathen. Temporal derivatives in the finite-element method on continuously deforming grids. SIAM J. Numer. Anal., 28:990-1003, 1991. doi: 10.1137/0728052.

B. J. P. Kaus, C. Steedman, and T. W. Becker. From passive continental margin to mountain belt: Insights from analytical and numerical models and application to Taiwan. Phys. Earth Planet. Int., 171:235-251, 2008. doi: 10.1016/j.pepi.2008.06.015.

B. J. P. Kaus, H. Muhlhaus, and D. A. May. A stabilization algorithm for geodynamic numerical simulations with a free surface. Phys. Earth Planet. Int., 181:12-20, 2010. doi: 10.1016/j.pepi.2010.04.007.

P. Koons. Two-sided orogen: collision and erosion from the sandbox to the Southern Alps, New Zealand. Geology, 18:679-682, 1990.

L. L. Lavier, W. Buck, and A. Poliakov. Factors controlling normal fault offset in an ideal brittle layer. J. Geophys. Res., 105:23431-23442, 2000.

B. P. Leonard. The ULTIMATE conservative difference scheme applied to unsteady onedimensional advection. Computer Methods in Applied Mechanics and Engineering, 88(1): 17-74, June 1991. ISSN 0045-7825. doi: 10.1016/0045-7825(91)90232-U.

L. Liu, S. Spasojevic, and M. Gurnis. Reconstructing Farallon plate subduction beneath North America back to the Late Cretaceous. Science, 322:934-938, 2008. doi: 10.1126/science.1162921. 
D May and L Moresi. Preconditioned iterative methods for Stokes flow problems arising in computational geodynamics. Physics of the Earth and Planetary Interiors, 171:33-47, 2008. doi: 10.1016/j.pepi.2008.07.036.

D. McKenzie. Surface deformation, gravity anomalies and convection. Geophysical Journal International, 48:211-238, 1977. doi: 10.1111/j.1365-246X.1977.tb01297.x.

H. J. Melosh and A. Raefsky. Dynamical origin of subduction zone topography. Geophys. J. Roy. Astr. Soc., 60:333-354, 1980.

P. Molnar and P. England. Late Cenozoic uplift of mountain ranges and global climate change: chicken and egg? Nature, 346:29-34, 1990.

H. Muhlhaus. Free surface modeling based on level sets. ICCEES, 3:225-232, 2007.

B. Parsons and S. Daly. The relationship between surface topography, gravity anomalies, and temperature structure of convection. J. Geophys. Res., 88:1129-1144, 1983. doi: 10.1029/JB088iB02p01129.

H. R. Poore, N. White, and S. Jones. A neogene chronology of iceland plume activity from V-shaped ridges. Earth Planet. Sci. Lett, 283:1-13, 2009. doi: 10.1016/j.epsl.2009.02.028.

H. Ramberg. Gravity, Deformation and the Earth's Crust: In Theory, Experiments and Geological Application. Academic Press, London and New York, 1967.

H. Schmeling, A. Y. Babeyko, A. Enns, C. Faccenna, F. Funiciello, T. Gerya, G.J. Golabek, S. Grigulla, B. J. P. Kaus, G. Morra, S. M. Schmalholz, and J. van Hunen. A benchmark comparison of spontaneous subduction models - towards a free surface. Phys. Earth Planet. Int., 171:198-223, 2008. doi: 10.1016/j.pepi.2008.06.028.

G. E. Shephard, R. D. Muller, L. Liu, and M. Gurnis. Miocene drainage reversal of the Amazon River driven by plate-mantle interaction. Nature Geoscience, 3:870-875, 2010. doi: 10.1038/NGEO1017.

S. C. Solomon, R. P. Comer, and J. W. Head. The evolution of impact basins Viscous relaxation of topographic relief. J. Geophys. Res., 87:3975-3992, 1982. doi: 10.1029/JB087iB05p03975.

S. Spasojevic, L. Liu, and M. Gurnis. Adjoint models of mantle convection with seismic, plate motion, and stratigraphic constraints: North America since the Late Cretaceous. Geochemistry Geophysics Geosystems, 10:24, 2009. doi: 2009 10.1029/2008GC002345.

E. Styles, D. R. Davies, and S. Goes. Mapping spherical seismic into physical structure: biases from 3-D phase-transition and thermal boundary-layer heterogeneity. Geophys. J. Int., 184:1371-1378, 2011. doi: 10.1111/j.1365-246X.2010.04914.x.

P. J. Tackley. Modelling compressible mantle convection with large viscosity contrasts in a three-dimensional spherical shell using the yin-yang grid. Phys. Earth Planet. Int., 171: 7-18, 2008. doi: 10.1016/j.pepi.2008.08.005. 
S. D. Willett. Orogeny and orography: The effects of erosion on the structure of mountain belts. J. Geophys. Res., 104:28957-28982, 1999. doi: 10.1029/1999JB900248.

C. R. Wilson. Modelling multiple-material flows on adaptive unstructured meshes. Ph.D Thesis, Imperial College London, 2009.

M. Wolstencroft, J. H. Davies, and D. R. Davies. Nusselt-Rayleigh number scaling for spherical shell Earth mantle simulation up to a Rayleigh number of $10^{9}$. Phys. Earth Planet. Int., 176:132-141, 2009. doi: 10.1016/j.pepi.2009.05.002.

J. D. Wright and K. G. Miller. Control of North Atlantic deep water circulation by the Greenland-Scotland Ridge. Paleoceanography, 11:157-170, 1996.

S. Zhong, M. Gurnis, and G. Hulbert. Accurate determination of surface normal stress in viscous flow from a consistent boundary flux method. Physics of the Earth and Planetary Interiors, 78:1-8, 1993. doi: 10.1016/0031-9201(93)90078-N.

S. Zhong, M. Gurnis, and L. Moresi. Free-surface formulation of mantle convection: I. basic theory and application to plumes. Geophys. J. Int, 127:708-718, 1996.

Shijie Zhong, Allen McNamara, Eh Tan, Louis Moresi, and Michael Gurnis. A benchmark study on mantle convection in a 3-D spherical shell using CitcomS. Geochemistry Geophysics Geosystems, 9:32 PP., 2008. doi: 2008 10.1029/2008GC002048. 
${ }_{630}$ Appendix B. Tables 


\begin{tabular}{llll}
\hline Mantle thickness & $D$ & $3 \times 10^{6}$ & $\mathrm{~m}$ \\
Viscosity & $\mu$ & $1 \times 10^{21}$ & $\mathrm{Pas}$ \\
Gravitational acceleration & $g$ & 10 & $\mathrm{~m} \mathrm{~s}^{-2}$ \\
Reference density & $\rho_{0}$ & 4500 & $\mathrm{~kg} \mathrm{~m}^{-3}$ \\
Top external density & $\left.\rho_{\text {ext }}\right|_{z>0}$ & 0 & $\mathrm{~kg} \mathrm{~m}^{-3}$ \\
Bottom external density & $\left.\rho_{\text {ext }}\right|_{z<-D}$ & 9000 & $\mathrm{~kg} \mathrm{~m}^{-3}$ \\
Thermal expansivity & $\alpha$ & $2 \times 10^{-5}$ & $\mathrm{~K}^{-1}$ \\
Temperature anomaly scale & $Q$ & 1 & $\mathrm{~K} \mathrm{~m}$ \\
\hline
\end{tabular}

Table B.1: Model parameters for the analytical test cases.

\section{Approximate}

Wavelength, Relaxation timescale, relaxation timescale (24),

\begin{tabular}{ccc}
$\lambda$ & $\tau(\mathrm{kyr})$ & $\tau_{0}(\mathrm{kyr})$ \\
\hline$D / 4$ & 11.81 & 11.81 \\
$D / 2$ & 5.90 & 5.90 \\
$D$ & 2.95 & 2.95 \\
$2 D$ & 1.52 & 1.48 \\
$4 D$ & 1.02 & 0.74 \\
\hline
\end{tabular}

Table B.2: Relaxation timescales for a single free surface.

\begin{tabular}{|c|c|c|c|}
\hline $\begin{array}{c}\text { Wavelength, } \\
\lambda\end{array}$ & $\begin{array}{l}\text { Relaxatio } \\
\tau_{+}(\mathrm{kyr})\end{array}$ & $\begin{array}{l}\text { timescales, } \\
\tau_{-}(\mathrm{kyr})\end{array}$ & $\begin{array}{c}\text { Approximate } \\
\text { relaxation timescale }(24), \\
\tau_{0}(\mathrm{kyr})\end{array}$ \\
\hline$D / 2$ & 5.90 & 5.90 & 5.90 \\
\hline$D$ & 3.03 & 2.87 & 2.95 \\
\hline $2 D$ & 2.04 & 0.98 & 1.48 \\
\hline $4 D$ & 1.89 & 0.15 & 0.74 \\
\hline
\end{tabular}

Table B.3: Relaxation timescales for two free surfaces. 
${ }_{631}$ Appendix C. Figures 


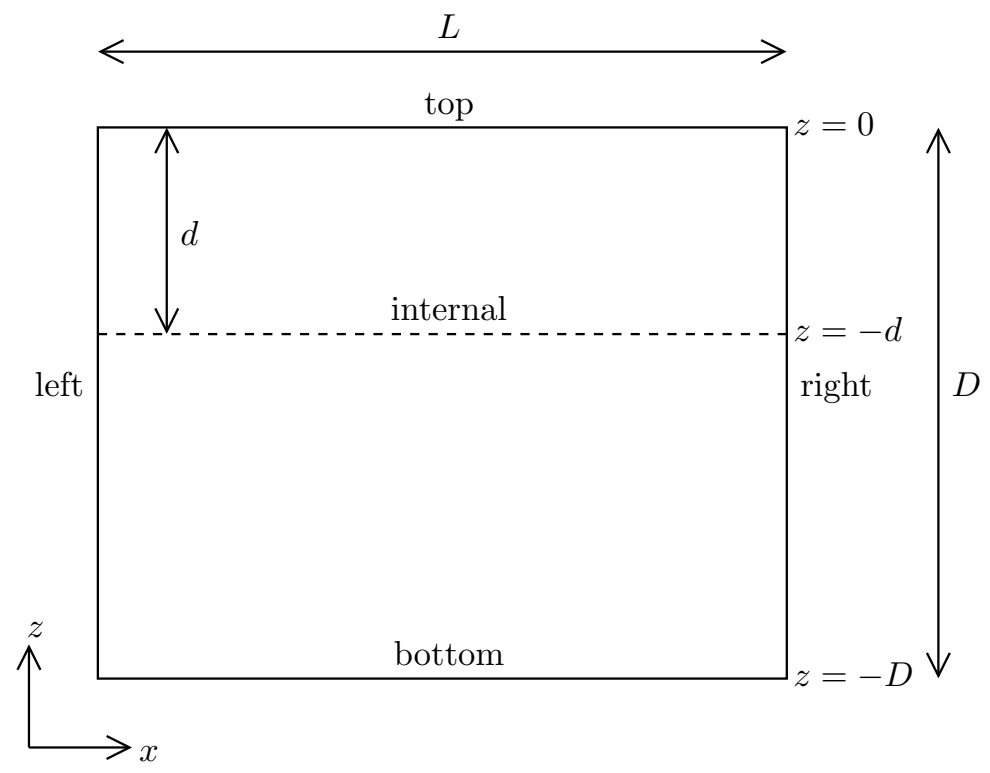

Figure C.1: Illustration of the idealized two-dimensional domain used in the analytical test cases. Boundary conditions are applied at the top, bottom, left and right boundaries while the internal surface may be used to apply a density anomaly. $z$ is taken as pointing upwards. 
(a) Temporal convergence

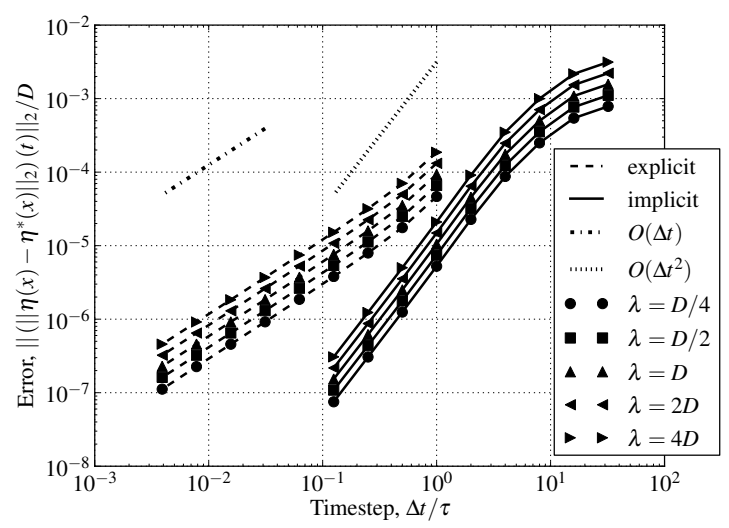

(b) Implicitness $(\lambda=D)$

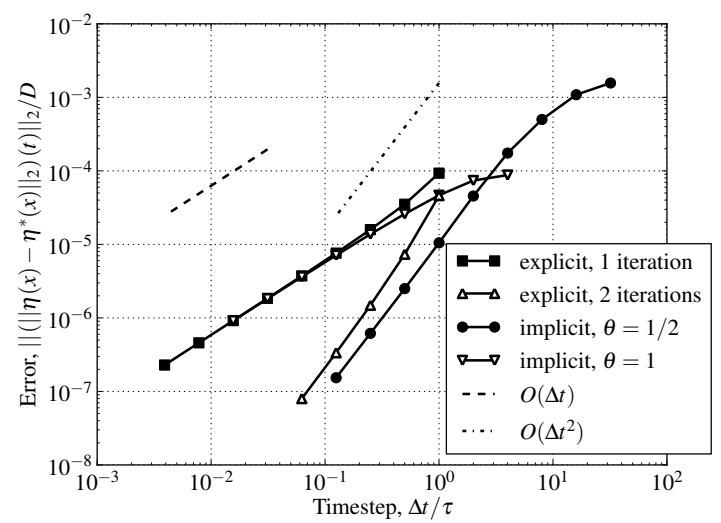

(c) Solution

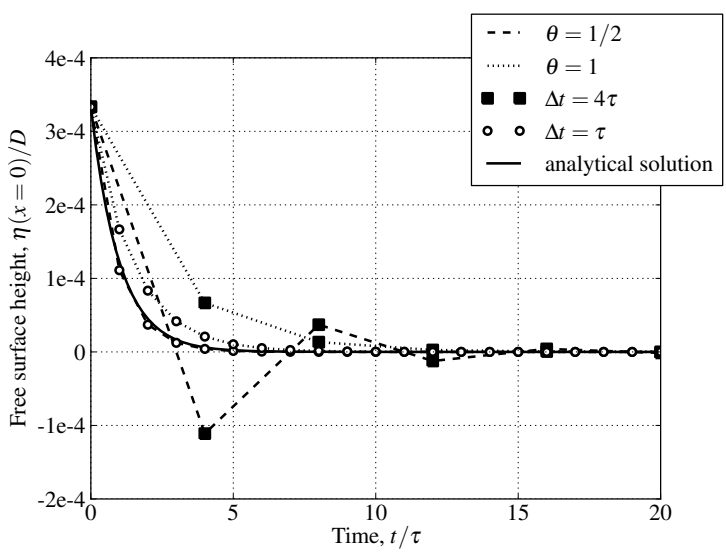

Figure C.2: (a) Error between the numerical, $\eta$, and analytical, $\eta^{*}$, free surface elevations versus timestep size, $\Delta t$, for the case with a single free surface. The error is normalized by domain depth, $D$, and measured both across the top of the domain and through time using an $L 2$ norm. The timestep is normalized by the free surface relaxation timescale, $\tau$ (see Table B.2). The explicit method is first order while the implicit method is second order for a range of wavelengths (domain widths), $\lambda$. (b) Normalized error in the free surface elevation versus normalized timestep for a wavelength equal to the domain depth, $\lambda=D$. The explicit method from (a) can be made second order by taking two iterations per timestep while the implicit method becomes first order when the implicitness factor, $\theta$, is set to one. In both (a) and (b) explicit simulations were run until $t=4 \tau$ while implicit simulations were run until $t=64 \tau$ before the temporal error was calculated. (c) The normalized free surface elevation at $x=0$ versus normalized time using the implicit method with different timesteps and $\theta$ s. The timesteps selected represent the cross-over in error in (b). At timesteps significantly larger than the relaxation time, $\theta=1 / 2$ results in oscillatory behavior and a smoother solution is achieved with $\theta=1$. At a timestep equal to the relaxation time, $\theta=1 / 2$ is almost indistinguishable from the analytical solution while with $\theta=1$ a larger error is still clearly present. All numerical solutions were calculated with a normalized grid resolution, $\Delta x / L=\Delta z / D$, of $1 / 80$. 
Temporal convergence

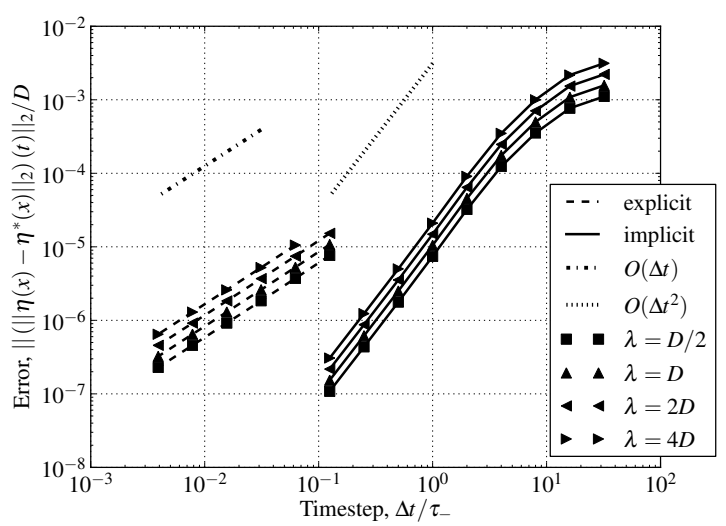

Figure C.3: Error between the numerical, $\eta$, and analytical, $\eta^{*}$, free surface elevations versus timestep size, $\Delta t$, for the case with two free surfaces. The error is normalized by domain depth, $D$, and measured both across the top of the domain and through time using an $L 2$ norm. The timestep is normalized by the minimum relaxation timescale, $\tau_{-}$(see Table B.3). The explicit method is first order accurate while the implicit method is second order accurate for a range of wavelengths (domain widths), $\lambda$. As both free surfaces are initialized with the same elevation the graph for the lower free surface is identical. All numerical solutions were calculated with a normalized grid resolution, $\Delta x / L=\Delta z / D$, of $1 / 80$. Explicit simulations were run until $t=4 \tau_{-}$while implicit simulations were run until $t=64 \tau_{-}$before the temporal error was calculated. 
(a) Temporal convergence

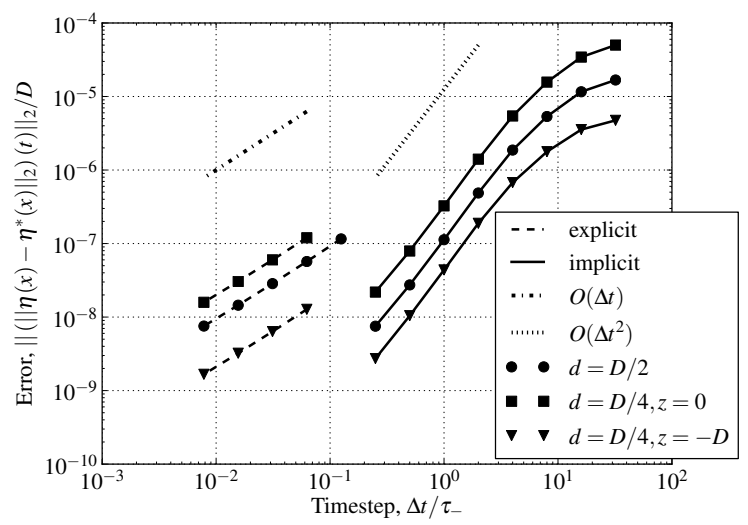

(b) Solution

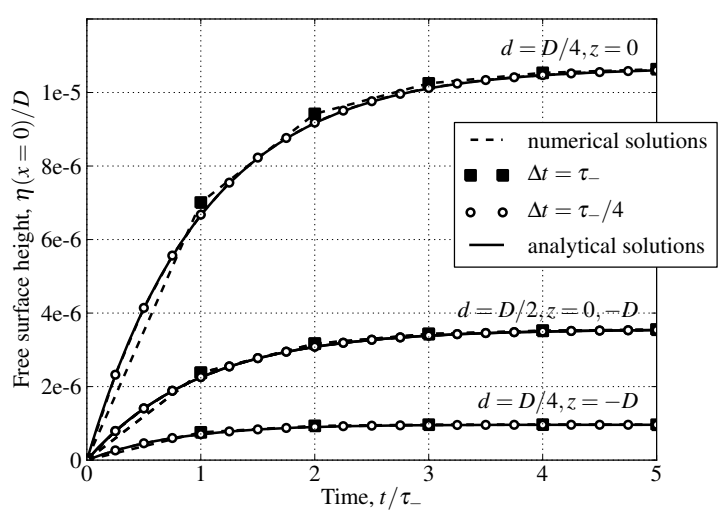

Figure C.4: (a) Error between the numerical, $\eta$ (or $\zeta$ for $z=-D$ ), and analytical, $\eta^{*}$ (or $\zeta^{*}$ for $z=-D$ ), free surface elevations versus timestep size, $\Delta t$, for the case with two free surfaces and a density anomaly. The error is normalized by domain depth, $D$, and measured both across the domain boundary and through time using an $L 2$ norm. The timestep is normalized by the minimum relaxation timescale, $\tau_{-}$(see Table B.3). The explicit method is first order while the implicit method is second order for a range of density anomaly depths, $d$, and for the upper, $z=0$, and lower, $z=-D$, free surfaces. For the $d=D / 2$ case the top and bottom errors are identical. Explicit simulations were run until $t=4 \tau_{\text {- }}$ while implicit simulations were run until $t=64 \tau_{-}$before the temporal error was calculated. (b) The normalized free surface elevation at $x=0(\eta(x=0) / D$ for $z=0, \zeta(x=0) / D$ for $z=-D)$ versus normalized time using the implicit method with different timesteps and anomaly depths, $d$. All numerical results were calculated using a normalized grid spacing, $\Delta x / L=\Delta z / D$, of $1 / 160$. 
(a) Spatial resolution dependence

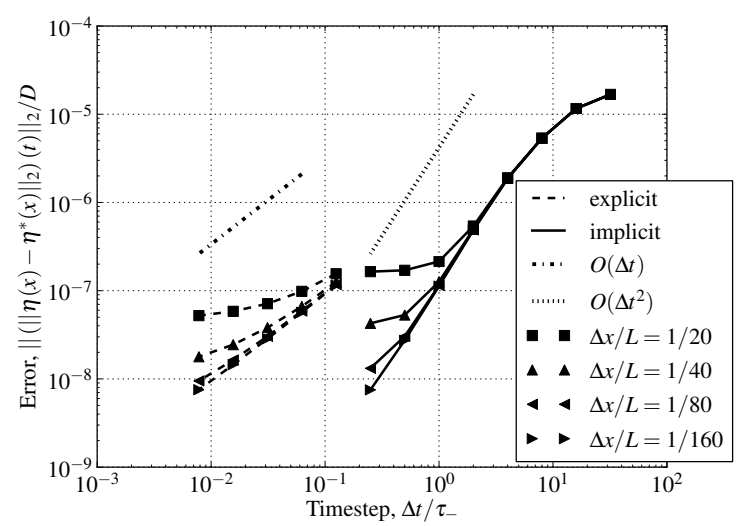

(b) Pressure solution $(x=\lambda / 2, d=D / 2)$

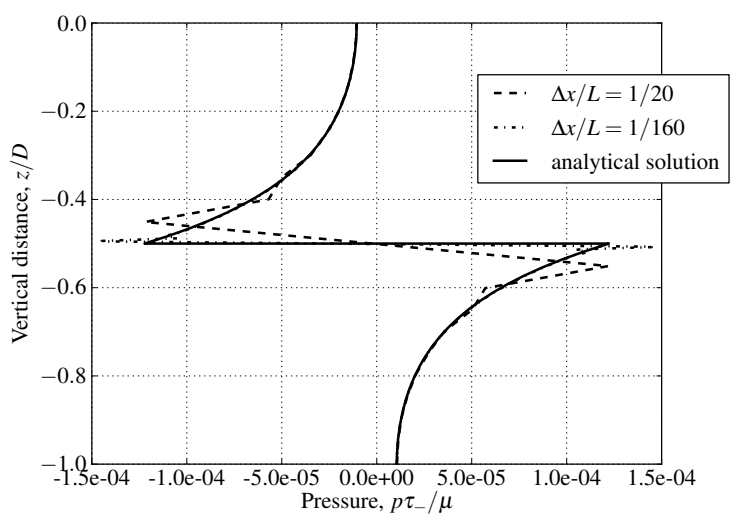

(c) Spatial convergence $(d=D / 2)$

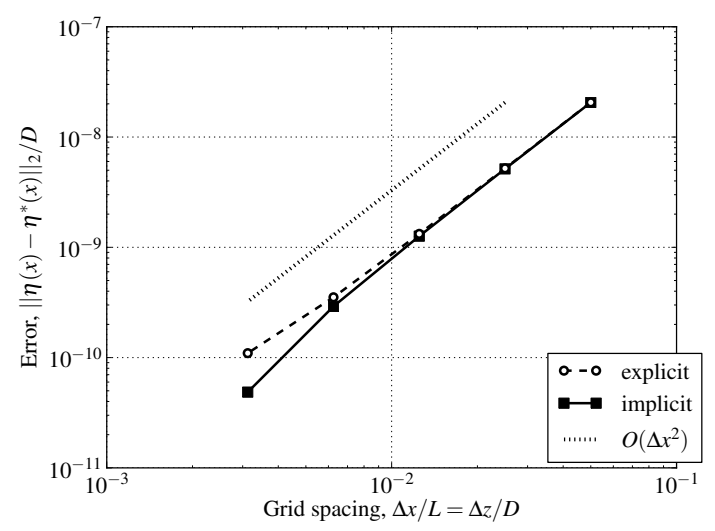

Figure C.5: (a) Error between the numerical, $\eta$, and analytical, $\eta^{*}$, free surface elevations versus timestep for the explicit and implicit methods at a range of spatial resolutions in the case with two free surfaces and a density anomaly. The meshes were refined in both horizontal and vertical direction, such that in all cases $\Delta x / L=\Delta z / D$. The error is normalized by domain depth, $D$, and measured both across the top of the domain and through time using an $L 2$ norm. The timestep is normalized by the minimum relaxation timescale, $\tau_{-}$(see Table B.3). High spatial resolution is necessary to ensure convergence to the correct analytical solution. Explicit simulations were run until $t=4 \tau_{-}$while implicit simulations were run until $t=64 \tau_{-}$before the temporal error was calculated. (b) A profile through the pressure solution at $x=\lambda / 2$ demonstrating the presence of a sharp discontinuity around the density anomaly, which must be adequately resolved. Numerical solutions are shown at two spatial resolutions after a time, $t$, of $64 \tau_{-}$. The analytical solution is at a steady state. (c) Normalized spatial error in the free surface elevation versus normalized grid spacing after the simulation has reached a steady state. Both implicit and explicit methods achieve second order accuracy using piecewise linear finite elements for pressure and the free surface and piecewise quadratic elements for velocity. All results were calculated using an anomaly depth, $d$, of half the domain depth, $D / 2$, and a wavelength equal to the domain depth, $\lambda=D$. 

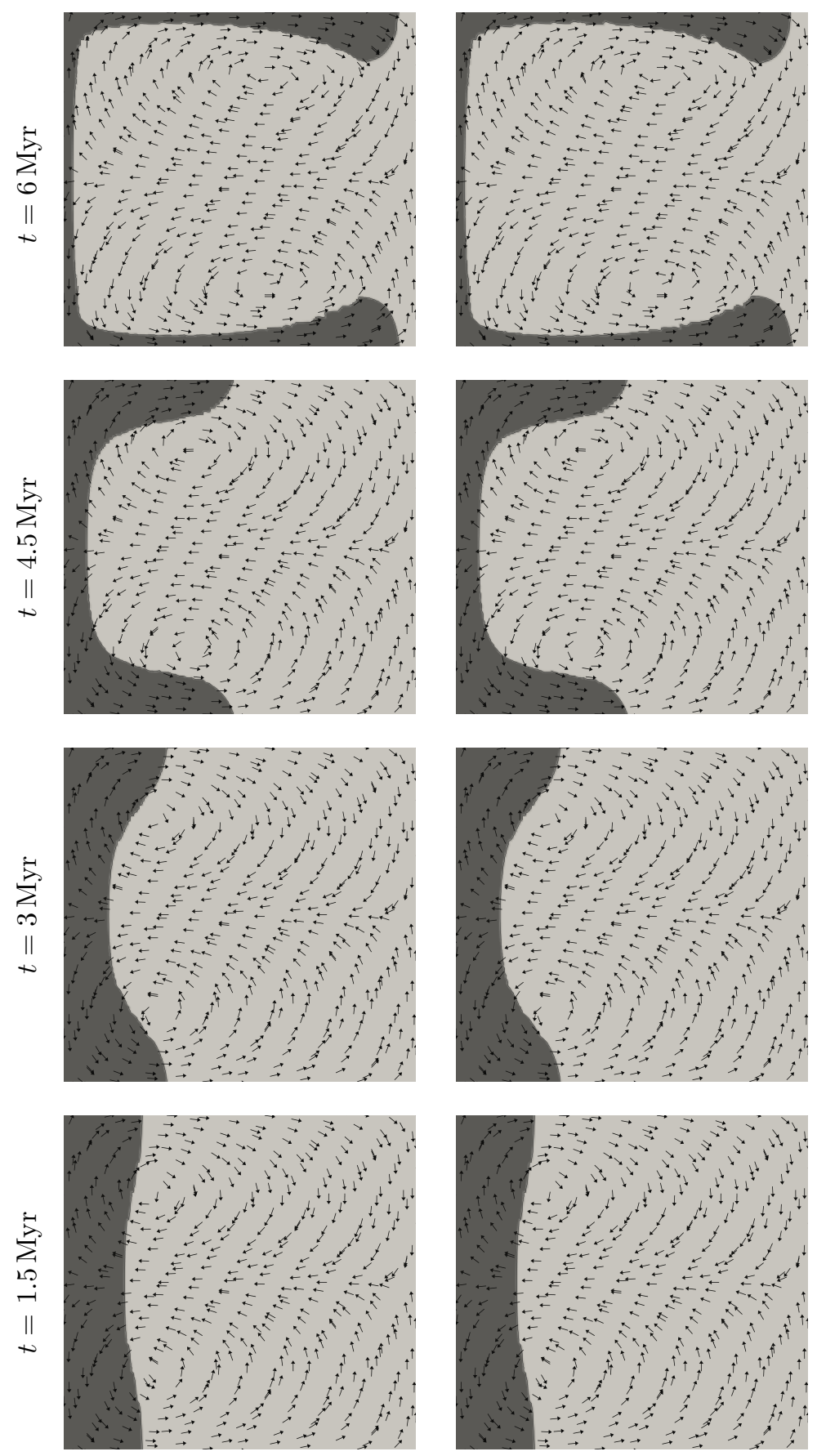

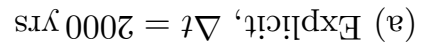
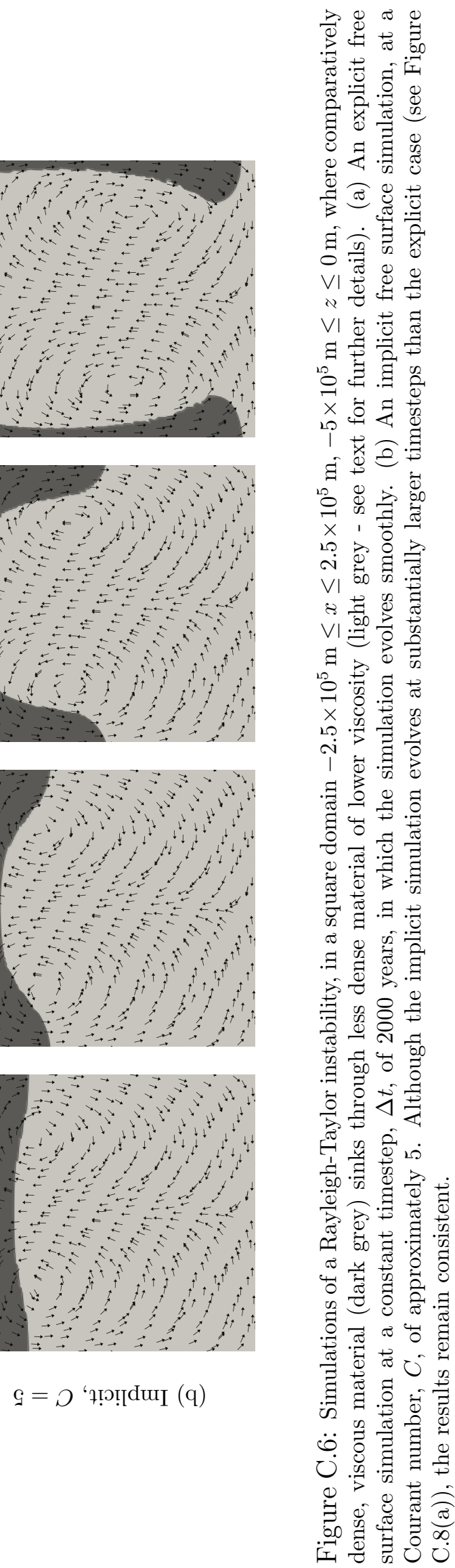

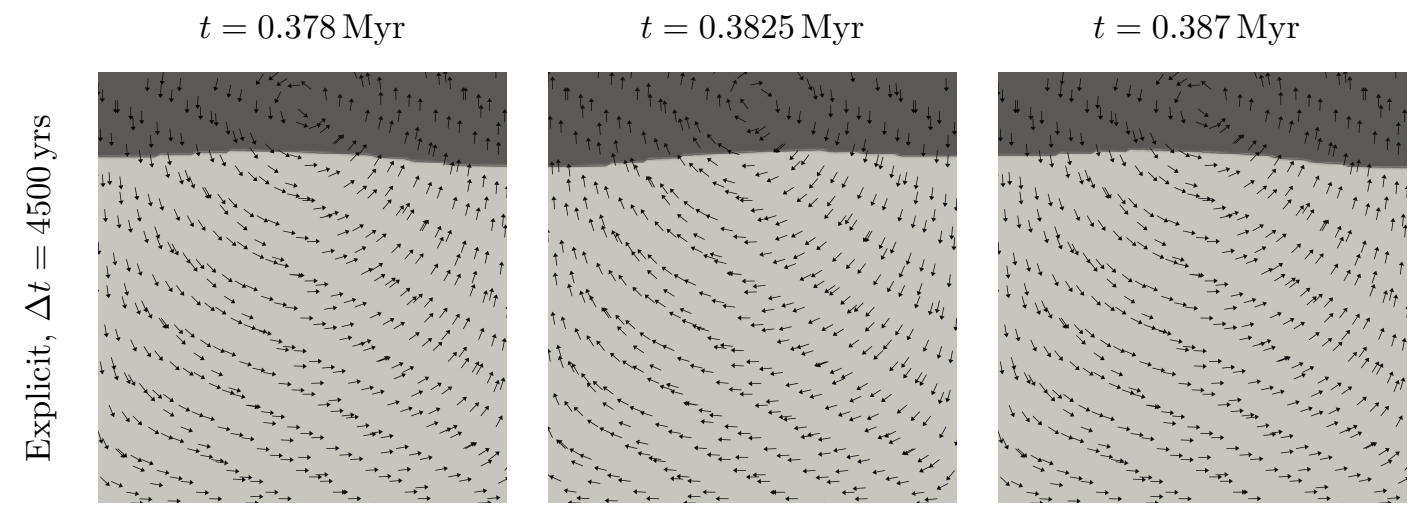

Figure C.7: Simulations of a Rayleigh-Taylor instability, in a square domain $-2.5 \times 10^{5} \mathrm{~m} \leq x \leq 2.5 \times 10^{5} \mathrm{~m}$, $-5 \times 10^{5} \mathrm{~m} \leq z \leq 0 \mathrm{~m}$, where comparatively dense, viscous material (dark grey) sinks through less dense material of lower viscosity (light grey - see text for further details). An explicit free surface simulation at a constant timestep, $\Delta t$, of 4500 years, in which a sloshing instability develops, where the velocity oscillates between consecutive timesteps. 
(a) Timestep size

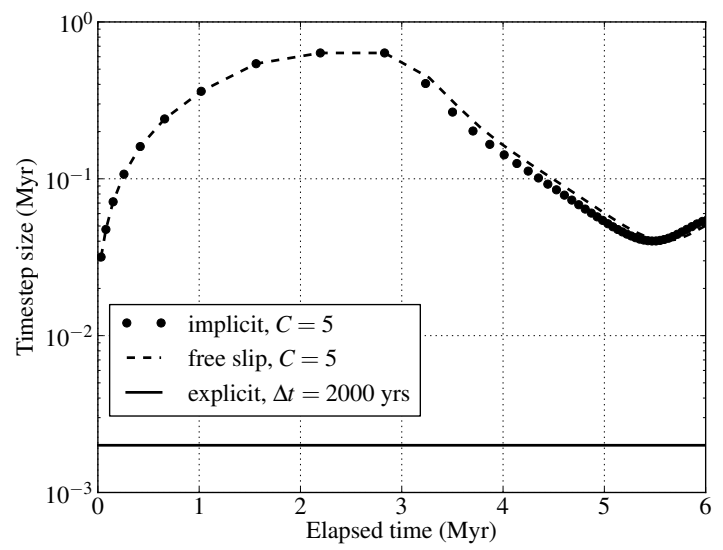

(b) Material interface depth

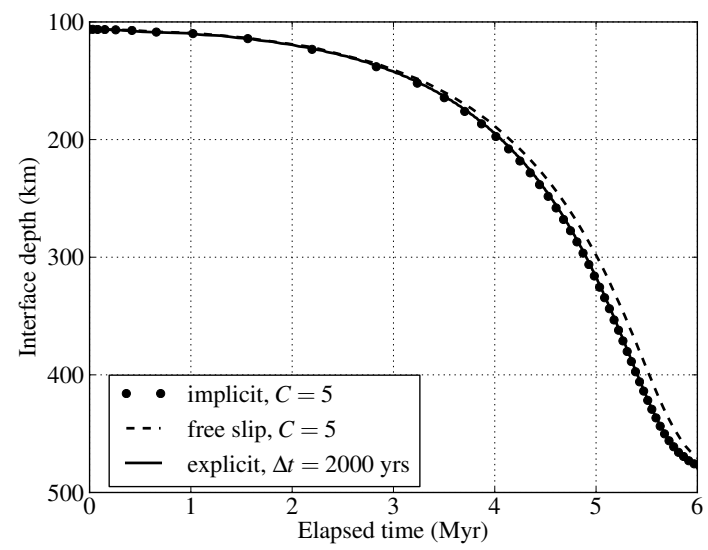

(c) Free surface height

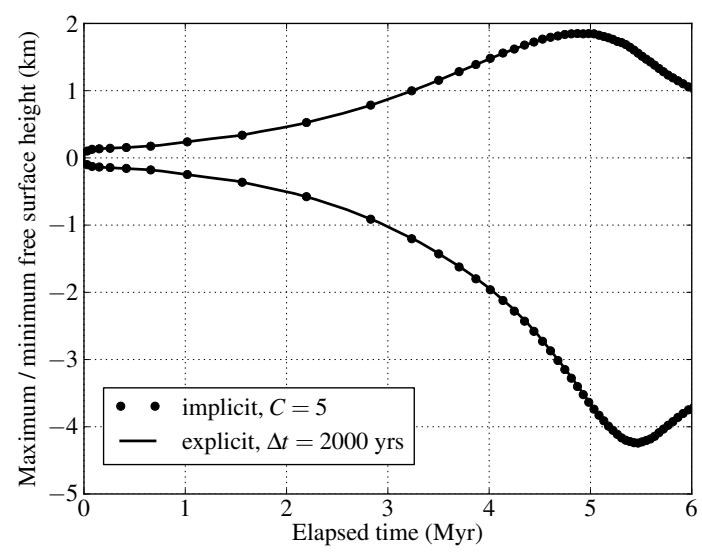

Figure C.8: A series of comparisons between explicit ( $\Delta t=2000$ years) and implicit (Courant number, $C=$ 5) free surface simulations of a Rayleigh Taylor instability in a square domain $-2.5 \times 10^{5} \mathrm{~m} \leq x \leq 2.5 \times 10^{5} \mathrm{~m}$, $-5 \times 10^{5} \mathrm{~m} \leq z \leq 0 \mathrm{~m}$ (see Figure C.6). (a) Timestep size, $\Delta t$, versus time - note the logarithmic scale. (b) Depth of the material interface at $x=-2.5 \times 10^{5} \mathrm{~m}$; (c) Maximum and minimum free surface height. For comparison, the results from a simulation with a free slip boundary condition on the top of the domain are shown. This targets the same Courant number as the simulation with an implicit free surface. Note that the implicit free surface simulation remains stable at substantially larger timesteps than the explicit simulation, while results remain consistent. It also evolves at timesteps similar to the free slip case. 